\title{
Fast three-dimensional black-blood MR imaging for carotid artery intra-plaque haemorrhage using DANTE-prepared FLASH (3D-DASH)
}

\author{
Linqing Li ${ }^{1}$, Luca Biasiolli ${ }^{2,3^{*}}$, Joshua T Chai ${ }^{3}$, Matthew D Robson ${ }^{2}$, Robin P Choudhury ${ }^{3}$, Ashok Handa ${ }^{4}$, \\ Peter Jezzard ${ }^{1}$
}

From 17th Annual SCMR Scientific Sessions

New Orleans, LA, USA. 16-19 January 2014

\section{Background}

DANTE (Delays Alternating with Nutation for Tailored Excitation) pulse trains are a rapid series of low flip angle RF pulses interspersed with gradients. We have previously demonstrated that when using DANTE pulse trains as a preparation module prior to imaging readout, the longitudinal magnetization of flowing spins is substantially attenuated, whereas the longitudinal magnetization of static tissue/fluid is mostly preserved [1]. In this study we introduce a new DANTE-prepared 3D FLASH T1 weighted (T1w) sequence (denoted '3DDASH') [2] that is able to generate $0.6 \mathrm{~mm}$ isotropic resolution images with an average imaging speed better than $2 \mathrm{sec} /$ slice.

\section{Methods}

6 healthy volunteers (males, 24 to 35 years) underwent (i) DIR (double inversion recovery)-prepared 2D-TSE, (ii) 3D-DASH and (iii) comparison MSDE prepared FLASH, 3D-MERGE, imaging[3]. 4 symptomatic patients (age range, 54-86) scheduled for carotid endarterectomy (> 70\% stenosis measured by ultrasound) underwent the same vessel wall imaging protocol. Written informed consent was obtained from all subjects. All scans were acquired using a 3T Siemens Verio scanner. A pair of dual-channel surface coils (Machnet, The Netherlands) were used. Cardiac gating was used for comparison DIR-prepared black blood scans. Protocol: axial imaging acquisition, identical 3D FLASH readout sequences for $3 \mathrm{D}-\mathrm{DASH}$ and 3D-MERGE, FOV $=150 \times 150 \mathrm{~mm}$, matrix size $256 \times 252$, interpolated to $512 \times 512$,

${ }^{2}$ Department of Medicine, University of Oxford, Oxford, UK Full list of author information is available at the end of the article partition thickness $=0.6 \mathrm{~mm}$, Number of averages $=2$, iPat $=2$, slices $=128$, FLASH flip angle $\alpha=10^{\circ}$, slice resolution $=63 \%$, phase and slice partial FT $=6 / 8$, Fat suppression $=$ water excitation-fast, TRinternal $=10 \mathrm{~ms}$, $\mathrm{BW}=130 \mathrm{~Hz} /$ pixel, resolution $=0.6 \mathrm{~mm}$ isotropic. Parameters for the DANTE module: flip angle (FA) $\alpha=$ $15^{\circ}$; Number of pulses $\mathrm{Np}=150$; time duration between DANTE pulses, $\mathrm{tD}=1 \mathrm{~ms} ; \mathrm{Gx}, \mathrm{y}, \mathrm{z}=20 \mathrm{mT} / \mathrm{m}$; gradient duration $\approx 1 \mathrm{~ms}$.

\section{Results}

Examples of the T1w image quality for the 3D-DASH sequence with $0.6 \mathrm{~mm}$ isotropic resolution versus the gold standard single-slice DIR-TSE sequence with slice thickness $2 \mathrm{~mm}$ are shown in Figure 1. The 3D-DASH scan acquisition time was 198 seconds, with $>6 \mathrm{~cm}$ coverage (128 slices). The hyper-intense signal on the T1w images indicates the presence of fresh intra-plaque haemorrhage (IPH) confirmed by histological examination shown in Figure 2. Compared with the current best 3D black blood (BB) technique (results were not shown), 3D-MERGE, 3D-DASH allows 75\%-100\% improvement in contrast-to-noise efficiency, CNReff.

\section{Conclusions}

3D-DASH is a promising new sequence for 3D isotropic $(0.6 \mathrm{~mm})$ fast black-blood T1 weighted imaging of the carotid arteries with high sensitivity to intra-plaque haemorrhage.

\section{Funding}

We thank the NIHR Oxford Biomedical Research Centre, BHF, and Dunhill Medical Trust for grant funding. 

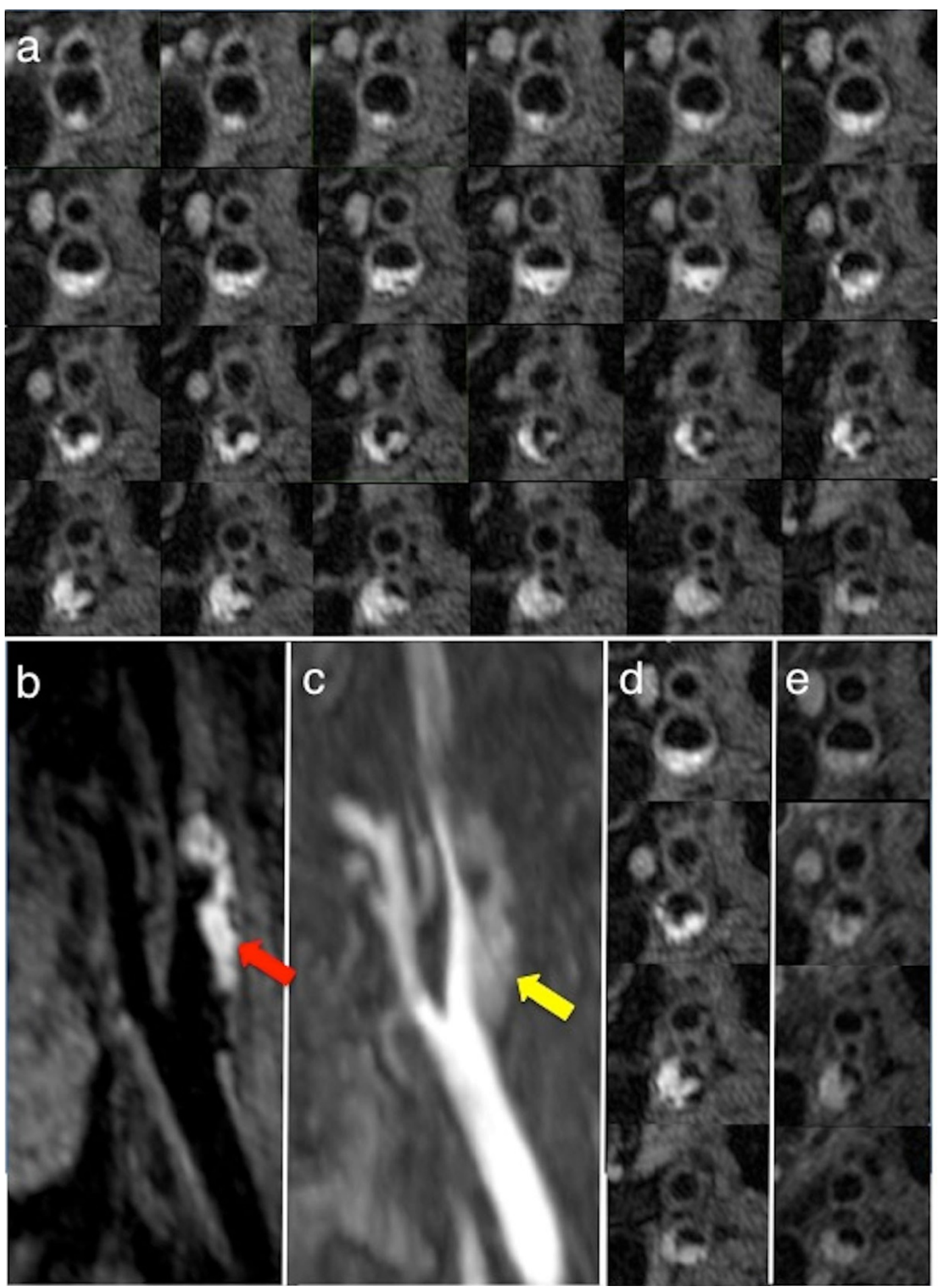

Figure $1 \mathrm{~T} 1 \mathrm{w}$ 3D-DASH images obtained from a patient with intra-plaque haemorrhage. a) 24 contiguous-slice whole plaque coverage from 3D-DASH images with isotropic $0.6 \mathrm{~mm}$ resolution. b) 3D-MPR sagittal view of the left carotid arteries reconstructed from the full 128-slice 3D-DASH dataset. c) 3D-MPR sagittal view reconstructed from the 3D-TOF data for comparison. d) Axial view slices of 3D-DASH and e) DIRprepared 2D-TSE images taken from the same slice positions for direct comparison. 


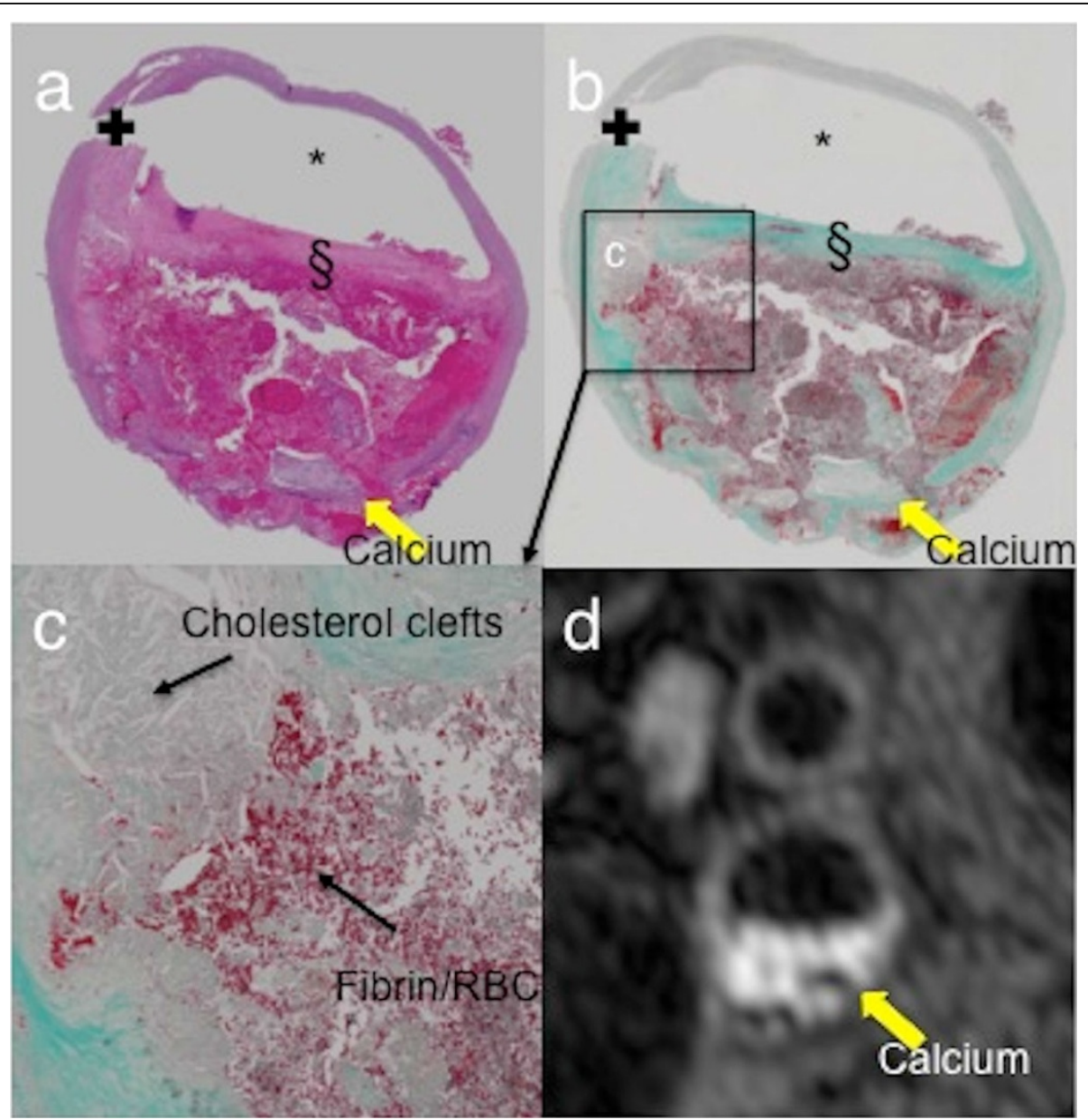

Figure 2 This histology figure confirms the presence of intra-plaque haemorrhage of the patient plaque in Fig. 4. a) Hematoxylin and eosin stain(H\&E). * lumen; $\S$ fibrous cap; +surgical artefacts. b) Maisson's trichrome staining of serial $5 \mu \mathrm{m}$ plaque sections. These images show a large lipid-rich necrotic core (LRNC) within an eccentric carotid atherosclerotic plaque with recent intra-plaque haemorrhage (IPH). c) Fibrin (in blood) stained bright pink in H\&E and bright red in trichrome. Background lipid core seen as cholesterol clefts. Collagen stained green in trichrome. Calcification stained blue by haematoxylin and remained pale/unstained in trichrome. d) Image taken from section location $6 \mathrm{~mm}$ above bifurcation.

\section{Authors' details}

${ }^{1}$ Department of Clinical Neurosciences, University of Oxford, Oxford, UK ${ }^{2}$ Department of Medicine, University of Oxford, Oxford, UK. ${ }^{3}$ Acute Vascular Imaging Centre, University of Oxford, Oxford, UK. ${ }^{4}$ Department of Surgery, University of Oxford, Oxford, UK.

Published: 16 January 2014

doi:10.1186/1532-429X-16-S1-075

Cite this article as: Li et al:: Fast three-dimensional black-blood MR imaging for carotid artery intra-plaque haemorrhage using

DANTE-prepared FLASH (3D-DASH). Journal of Cardiovascular Magnetic Resonance 2014 16(Suppl 1):O75.

\section{Submit your next manuscript to BioMed Central and take full advantage of:}

- Convenient online submission

- Thorough peer review

- No space constraints or color figure charges

- Immediate publication on acceptance

- Inclusion in PubMed, CAS, Scopus and Google Scholar

- Research which is freely available for redistribution 